\title{
Panorama Setorial e Perspectivas na Área de Polímeros Biodegradáveis
}

\author{
Daniele M. B. Falcone, José Augusto M. Agnelli \\ Departamento de Engenharia de Materiais, UFSCar \\ Leandro I. L. de Faria \\ Departamento de Ciência da Informação, UFSCar
}

\begin{abstract}
Resumo: Neste trabalho, envolvendo polímeros biodegradáveis, buscou-se obter indicadores por meio da análise de patentes para avaliar as perspectivas e oportunidades de atuação da área de polímeros. Utilizou-se para tal a base de dados Espacenet e o software Vantage Point. São matéria desse estudo os polímeros: poli(hidroxibutirato) - PHB, poli(hidroxibutirato-cohidroxivalerato) - PHBV, poli(ácido lático) - PLA, poli(e-caprolactona) - PCL e os polihidroxialcanoatos (PHAs), tratados mais detalhadamente por serem a classe geral dos poliésteres microbiais. Verificou-se que a área de polímeros biodegradáveis, apesar de recente e em desenvolvimento, apresenta grande potencial mediante o panorama atual de consumo dos materiais poliméricos. Observou-se, de uma forma geral, uma grande diversidade de temas e oportunidades de estudo em compostos, blendas, biodegradação e aplicações.
\end{abstract}

Palavras-chave: Polímeros biodegradáveis, polihidroxialcanoatos, poli(hidroxibutirato), poli(hidroxibutirato-co-hidroxivalerato), poli(E-caprolactona).

\section{Biodegradable Polymers: Sectorial Overview and Prospects}

Abstract: This work on biodegradable polymers involved an analysis of patents to identify indicators for evaluating the prospects and opportunities of action in the field of polymers. We used the Spacenet database and Vantage Point software. The study encompassed the following polymers: polyhydroxybutyrate - PHB, polyhydroxybutyrate-co-hydroxyvalerate - PHBV, polylactic acid - PLA, poly ( $\varepsilon$-caprolactone) - PCL and the polyhydroxyalkanoates (PHAs), dealt in greater detail because they represent the general class of microbial polyesters. We found that, although the field of biodegradable polymers is new and still under development, it holds great potential in view of present widespread use of polymeric materials. Overall we found a great diversity of themes and opportunities for studies on compounds, blends, biodegradation and applications.

Keywords:Biodegradablepolymers, polyhydroxyalkanoates, polyhydroxybutyrate, polyhydroxybutyrate-co-hydroxyvalerate, poly (E-caprolactone).

\section{Introdução}

Nos dias atuais, os polímeros biodegradáveis vêm se destacando cada vez mais. Busca-se um material com durabilidade em uso e degradabilidade após o descarte. Estes materiais se constituem em compostos que devido à ação de microorganismos (fungos e bactérias) e macroorganismos serão degradados a compostos de baixa massa molar ${ }^{[1]}$. Outra característica importante refere-se ao fato dos mesmos serem provenientes de fontes renováveis. Tais materiais encontraram aplicações na área médica (fios de sutura, implantes, sistema de liberação controlada de drogas, enxerto vascular, etc) em decorrência de sua biocompatibilidade, capacidade de dissolução no interior dos organismos e propriedades mecânicas adequadas a tais aplicações. Com o tempo, poderão também encontrar aplicações no setor de embalagens e setor agrícola ${ }^{[2]}$. Este fato apresenta grande interesse, uma vez que o setor de embalagens é responsável por mais de um terço do total de resinas transformadas no Brasil ${ }^{[3]}$. No ano de 2003 a produção anual de plásticos no Brasil foi correspondente a 2,3 milhões de toneladas, sendo que $40 \%$ destinava-se à indústria de embalagens ${ }^{[4-6]}$. Atualmente, a situação mostra um aumento no consumo de plásticos que no futuro serão dispostos no ambiente acarretando problemas ecológicos. Por outro lado, os polímeros biodegradáveis ainda não são capazes de competir com os polímeros tradicionais, especialmente devido ao custo elevado e desempenho inferior. Estes polímeros chegam a custar quatro vezes mais que os polímeros tradicionais. Em consequiência, o interesse por estes materiais aliado à maior preocupação ambiental levou a um expressivo aumento nas investigações para o uso dos mesmos. Os principais polímeros biodegradáveis são poliésteres baseados nos ácidos hidroxi-carbônicos. Entre eles pode-se mencionar: PHB - poli(hidroxibutirato); PHBV - poli(hidroxibutirato-cohidroxivalerato); PLA - poli(ácido lático); PCL - poli( $\varepsilon$ caprolactona). Tanto o PHB, como o PHBV pertencem à 
família dos polihidroxialcanoatos (PHAs), que se enquadram na classe dos poliésteres microbiais.

Assim sendo, este trabalho teve como objetivo avaliar as perspectivas e oportunidades de atuação relacionadas aos polímeros biodegradáveis, utilizando-se de indicadores, por meio da análise de patentes. Para os PHAs foi efetuada um estudo mais detalhado.

\section{Metodologia}

\section{Utilização da base de dados Espacenet}

Para a obtenção dos dados a serem analisados foi utilizada a base de dados Espacenet, base disponível mais adequada quando da realização da pesquisa. A busca foi efetuada através de três procedimentos:

1. Busca por tipo de polímero + código de classificação internacional: (polyhydroxyalkanoat* or hydroxyalkanoat* or pha) and C, (polyhydroxybutyrat* or hydroxybutyrat* or phb) and C, (polyhydroxybutyrat* or hydroxybutyrat* or phb) and C (polyhydroxyvalerat* or hydroxyvalerat* or phv) and C (polylacti* or poly(lactic acid) or lactic acid polymer or pla) and C (polycaprolacton* or caprolacton* or pcl) and C.

O código de classificação $C$ corresponde a química e metalurgia e foi utilizado para concentrar a busca nas aplicações de polímeros biodegradáveis.

2. Busca por tipo de material + código de classificação internacional: (biodegrad* and polymer*) and (C08G63 or C08L67).

O código de classificação internacional C08G63 corresponde a compostos macromoleculares obtidos por reações formando uma ligação éster carboxílica na cadeia principal da macromolécula e o código C08L67 corresponde a composições de poliésteres obtidos por reações formando uma ligação éster carboxílica na cadeia principal e composições de derivados de tais polímeros. Estes códigos foram utilizados para a busca, pois se mostraram importantes para a área de polímeros biodegradáveis.

3. Busca por detentor da patente + código: (Monsanto or Metabolix or Procter or Gamble) and (C08G63 or C08L67).

Utilizou-se estas empresas durante a realização da pesquisa, pois foram mencionadas em documentos estudados previamente como empresas que se destacam em patenteamento na área de polímeros biodegradáveis.

Estes resultados foram compilados e posteriormente analisados utilizando-se o software Vantage Point. Em virtude da base de dados utilizada, a análise baseou-se no título e no código de classificação. Conseqüentemente, para uma visualização geral do número de patentes versus tipo de polímero foi feita uma subdivisão em 7 categorias: PHA, PHB, PHBV, PLA, PCL, biodegradável, geral. O grupo biodegradável relacionou-se às patentes que não apresentaram no título um polímero específico, mas sim o termo mais genérico, e tam- bém às patentes que envolviam dois ou mais polímeros dos que estão em estudo (por exemplo: blenda de um PHA com PLA). No grupo geral foram incluídas as patentes com títulos mais gerais, onde mesmo através do código de classificação não foi possível enquadrá-las como biodegradáveis (podendo estar ou não relacionadas a polímeros biodegradáveis). Foi realizado também, um estudo mais detalhado para a classe dos PHAs, subdividindo as patentes de acordo com o código de classificação, em patentes relacionadas com obtenção, processos para a formação de misturas e aplicações (aplicações da área médica e aplicações gerais).

\section{Resultados e Discussão}

Inicialmente serão apresentados os resultados mais gerais, e posteriormente os resultados obtidos para os polímeros em questão. Através da Figura 1, tem-se um panorama geral relacionado ao número de patentes em função do ano de depósito. Pode-se observar que houve um crescimento expressivo na quantidade de patentes relacionadas a polímeros biodegradáveis a partir da década de 90 , em virtude da maior preocupação em matéria ambiental e da legislação presente em alguns países.

Em relação ao código de classificação internacional, encontrou-se 65 classificações diferentes, sendo que destas foram selecionadas as 10 com maior número de ocorrências. Na Tabela 1 pode-se observar os códigos de classificação em função do ano de depósito assim como as descrições dos respectivos códigos de classificação.

Através dos dados apresentados na Tabela 1, pôde-se verificar que a maior parte das patentes estão relacionadas com misturas, ou seja, composições (C08L), processos para a formação de misturas (C08J) e ingredientes de composições $(\mathrm{C} 08 \mathrm{~K})$. Posteriormente vêm as patentes relacionadas a obtenção dos polímeros (C08G, C12P, C08F). É importante mencionar que o número de patentes relacionadas com aplicações (A61K, B32B) é pequeno. Esses dados tornaram evidente que a área de polímeros biodegradáveis é um campo ainda em desenvolvimento.

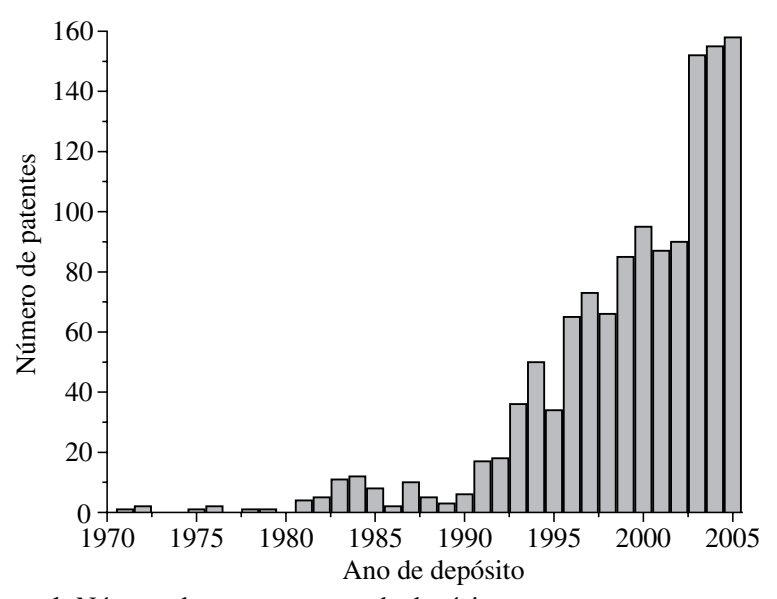

Figura 1. Número de patentes $x$ ano de depósito. 
Falcone, D. M. B. et al. - Panorama setorial e perspectivas na área de polímeros biodegradáveis

Tabela 1. Código de classificação vs. ano de depósito e descrição dos códigos de classificação.

\begin{tabular}{lcrrrrrrrrrr}
\hline Ano de depósito & C08G & C08L & C08J & C12P & C08K & C12N & B29C & A61K & B32B & C08F \\
\hline $1971-1993$ & 63 & 44 & 11 & 12 & 11 & 6 & 3 & 16 & $*$ & 9 \\
1994 & 25 & 9 & 2 & 7 & 3 & 5 & 5 & $*$ & 1 & 1 \\
1995 & 15 & 9 & 3 & 4 & 1 & 3 & 2 & 2 & 2 & $*$ \\
1996 & 36 & 22 & 10 & 7 & 5 & 1 & 3 & 1 & 2 & 1 \\
1997 & 27 & 31 & 9 & 11 & 11 & 2 & 2 & $*$ & 1 & 1 \\
1998 & 40 & 14 & 5 & 6 & 9 & 2 & $*$ & 1 & 1 & 3 \\
1999 & 35 & 28 & 5 & 16 & 5 & 9 & 5 & 1 & 1 & 2 \\
2000 & 31 & 15 & 13 & 18 & 7 & 11 & 8 & 1 & 4 & 2 \\
2001 & 26 & 20 & 23 & 17 & 7 & 11 & 10 & 2 & 7 & 3 \\
2002 & 35 & 21 & 19 & 20 & 5 & 7 & 9 & 4 & 4 & $*$ \\
2003 & 66 & 44 & 28 & 31 & 10 & 11 & 14 & 6 & 3 & 4 \\
2004 & 37 & 68 & 35 & 18 & 31 & 11 & 16 & 10 & 7 & 4 \\
2005 & 39 & 65 & 33 & 22 & 43 & 14 & 15 & 8 & 8 & 2 \\
Total & 475 & 390 & 196 & 189 & 148 & 93 & 92 & 52 & 41 & 32 \\
\hline
\end{tabular}

C08G Compostos macromoleculares obtidos por reações outras que não envolvendo ligações insaturadas carbono a carbono.

C08L Composições de compostos macromoleculares.

C12P Processos de fermentação ou utilizando enzimas para sintetizar um composto ou uma composição química desejada ou para separar isômeros óticos de uma mistura racêmica.

C08J Elaboração, processos gerais para formar misturas, pós-tratamento não abrangido pelas subclasses C08B, C08C, C08F, C08G.

C08K Emprego de substâncias inorgânicas ou orgânicas não macromoleculares como ingredientes de composições.

C12N Microorganismos ou enzimas, suas composições, propagação, preservação, ou manutenção de microorganismos ou tecidos, engenharia genética ou de mutações, meios de cultura.

B29C Modelagem ou união de materiais plásticos, modelagem de substâncias em estado plástico em geral, pós-tratamento dos produtos modelados.

A61K Preparados para finalidades médicas, odontológicas ou higiênicas.

B32B Produtos em camadas, isto é, produtos estruturados com camadas de forma plana, p. e., em forma celular ou alveolar.

C08F Compostos macromoleculares obtidos por reações compreendendo apenas ligações insaturadas carbono a carbono.

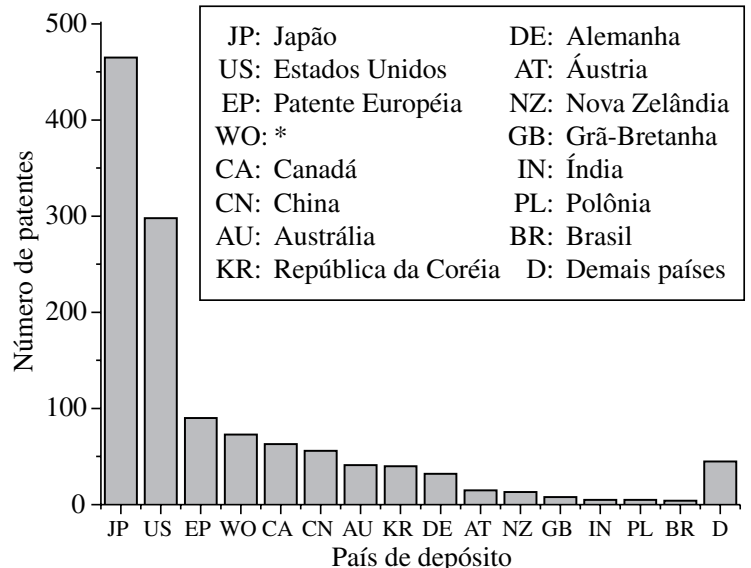

Figura 2. Número de patentes $x$ país de depósito. *(WO): pedido de patentes feito por um mecanismo chamado PCT através do qual o depósito é feito simultaneamente em vários países, sendo que em cada país a patente recebe um número diferente.

Também é importante avaliar o número de patentes em função do país de depósito, sendo estes dados ilustrados no Figura 2.
Os dados obtidos mostram que Estados Unidos e Japão detêm o maior número de patentes nesta área, sendo que os Estados Unidos é o país com leis mais rígidas em relação aos materiais ambientalmente aceitáveis, apresentando leis de reciclagem, leis que requerem negócios comerciais para separar os recicláveis, etc ${ }^{[7]}$. Pode-se dizer que embora a Alemanha não esteja entre os países que possuem o maior número de patentes na área em estudo, incentiva a utilização dos materiais ambientalmente corretos ao lado dos Estados Unidos e Japão. Isto posto, mostra que os países desenvolvidos se destacam em relação à preocupação ecológica preferindo pagar um pouco mais por um produto a fim de preservar o meio ambiente. Por possuir uma empresa atuando nesta área (Basf), a Alemanha, mesmo incentivando a utilização destes materiais possui poucas patentes. O Brasil, apresenta quatro patentes na área de polímeros biodegradáveis de acordo com a pesquisa realizada.

Em relação aos detentores das patentes, encontrou-se cerca de 357 empresas com patentes nesta área das quais foram selecionadas aquelas que se destacaram, Tabela 2.

Pode-se observar que além das três empresas patenteadoras importantes nesta área (Procter \& Gamble, Monsanto 
e Metabolix) indentificou-se também outras empresas muito fortes em P\&D neste ramo (Shimadzu, Canon, Kanebo, entre outras). Vale ressaltar que a maneira como a busca foi efetuada, utilizando-se do nome das três empresas pode ter interferido na ordem em que as mesmas aparecem no ranking.

Após esta análise mais geral foi realizada uma análise voltada aos polímeros biodegradáveis, subdividindo os títulos obtidos em 7 categorias: PHA, PHB, PHBV, PLA, PCL, biodegradável, geral, como já explicado na metodologia. A Tabela 3 mostra o número de patentes em relação ao tipo de polímero.

Entre os polímeros biodegradáveis estudados, o PLA se destacou por possuir o maior número de patentes. Isto em decorrência de ser disponibilizado em indústrias com tradição em síntese química e patenteamento. Após toda esta análise foi feito também um estudo mais específico para os PHAs,

Tabela 2. Detentor da patente $x$ número de patentes.

\begin{tabular}{lc}
\hline \multicolumn{1}{c}{ Detentor } & Número de patentes \\
\hline Shimadzu & 87 \\
Procter\&Gamble & 86 \\
Monsanto & 64 \\
Metabolix & 61 \\
Canon & 49 \\
Kanebo & 45 \\
Mitsubishi Plastics & 44 \\
Basf & 33 \\
Unitika & 33 \\
Toray Industries & 31 \\
Mitsui Chemical Inc & 25 \\
Korea Inst Sicence Tech & 23 \\
Daicel Chem & 21 \\
ICI PLC & 19 \\
Du Pont & 14 \\
Agency Ind Science Techn & 12 \\
Toyo Boseki & 11 \\
\hline
\end{tabular}

Tabela 3. Número de patentes $x$ tipo de polímero.

\begin{tabular}{cc}
\hline Tipo de polímero & Número de patentes \\
\hline PHA & 312 \\
PLA & 523 \\
PCL & 85 \\
PHB & 58 \\
PHBV & 12 \\
Biodegradável & 240 \\
Geral & 25 \\
\hline
\end{tabular}

Tabela 4. Fração de patentes dos PHAs relacionadas a obtenção, processos para a formação de misturas e aplicações.

\begin{tabular}{lc}
\hline \multicolumn{1}{c}{ Grupos } & $\begin{array}{c}\text { Fração de } \\
\text { patentes (\%) }\end{array}$ \\
\hline Obtenção & 69,6 \\
Processos para a formação de misturas & 5,4 \\
Aplicações (área médica) & 5,4 \\
Aplicações (gerais) & 3,5 \\
\hline
\end{tabular}

conforme mencionado na metodologia. Na Tabela 4, pode-se visualizar a porcentagem de patentes para cada grupo (obtenção, processos para a formação de misturas e aplicações) para os PHAs.

A maior parte das patentes está relacionada à obtenção dos PHAs. Provavelmente em virtude desta área ser mais recente e também pelo fato dos PHAs serem obtidos tanto através da síntese bacterial como através da síntese química.

Na Tabela 5 são mostrados os códigos de classificação relacionados com obtenção, processos para a formação de misturas e aplicações.

Tabela 5. Códigos de classificação relacionados com obtenção (C08G- C07B), processos para a formação de misturas (C08J) e aplicações (A61K- A61F).

\begin{tabular}{ll}
\hline \multicolumn{2}{c}{ Classificação } \\
\hline C08G & Compostos macromoleculares obtidos por \\
& reações outras que não envolvendo ligações \\
& insaturadas carbono a carbono.
\end{tabular}

C12P Processos de fermentação ou utilizando enzimas para sintetizar um composto ou uma composição química desejada ou para separar isômeros óticos de uma mistura racêmica.

C08F Compostos macromoleculares obtidos por reações compreendendo apenas ligações insaturadas carbono a carbono.

$\mathrm{A} 10 \mathrm{H}$ Plantas novas.

C12M Aparelhagem para enzimologia ou microbiologia, aparelhagem para cultura de algas unicelulares, células de plantas ou animais, tecidos ou vírus.

C07B Métodos gerais de química orgânica, aparelhos para os mesmos.

C08J Elaboração, processos gerais para formar misturas, pós-tratamento não abrangido pelas subclasses C08B, C08C, C08F, C08G.

A61K Preparados para finalidades médicas, odontológicas ou higiências.

B32B Produtos em camadas, isto é, produtos estruturados com camadas de forma plana, p. e., em forma celular ou alveolar.

A61L Métodos ou aparelhos para esterilizar materiais ou objetos em geral, desinfecção, esterilização do ar, aspectos químicos de ataduras, curativos, ou artigos cirúrgicos.

C09D Tintas de escrever ou de imprimir, tintas para pintar, vernizes, lacas, corantes para madeira, removedores químicos de tinta, pastas ou sólidos para colorir ou imprimir.

C09J Emprego de outras substâncias que não a cola, como adesivos, processos adesivos em geral, parte não mecânica.

A61F Prótese, aparelhos ortopédicos ou de enfermagem, dispositivos anticoncepcionais, fomentação, tratamento ou proteção dos olhos ou ouvidos, bandagens. 


\section{Conclusões}

Através dos resultados obtidos pôde-se perceber o panorama atual dos polímeros biodegradáveis mostrando um campo em desenvolvimento, com crescente utilização de polímeros nos mais variados setores. É uma área de grande potencial de estudos para viabilização do seu uso. Todos os polímeros em estudo mostraram-se muito interessantes para serem investigados, especialmente o PHB, por ser um polímero produzido no Brasil. A diversidade em temas abrangidos é grande (composições, blendas, copolímeros, obtenção, separação, aplicações gerais e médicas, entre outras) sendo, entretanto, a maior parte das patentes relacionadas com misturas e obtenção e poucas patentes relacionadas com aplicações. É válido atentar para o fato de que a análise efetuada através da Espacenet, permite a utilização apenas do título da patente, podendo tal fato ter se tornado uma fonte de erro neste trabalho. Os resultados obtidos mostram-se como referenciais, sendo necessário para um estudo mais completo utilizar-se também de outras bases de dados e outras fontes.

\section{Agradecimentos}

Os autores agradecem ao $\mathrm{CNPq}$ pelo suporte financeiro.

\section{Referências Bibliográficas}

1. Karlsson, S. \& Albertson, A. C. - Polymer Engineering and Science, 38, p.1251 (1998).

2. Chandra, R. \& Rustgi, R. - Progress in Polymer Science, 23, p.1273, (1998).

3. VD Plast. Nobreza do plástico. Jul. 2003. Disponível em: http://www.vdplast.com.br/artigo_01072003.asp. Acesso em: 16 dez. (2004).

4. Sotero, A. P. - Plásticos Biodegradáveis trazem melhoria ambiental. Ago. (2000). Diponível em: <http://www.jorplast. com.br/jpago00/ago006.html>. Acesso em: 06 jan. (2004).

5. Embanews, São Paulo, Brasil, n. 163, p. 34, 2004.

6. O Plástico no Brasil 2004, Plásticos em Revista, São Paulo, n. 495, jul. (2004).

7. Narayan, R. - "Impact of Governmental Policies, Regulations, and Standards Activities on an Emerging Biodegradable Plastics Industry", in: Proceedings of the Third International Scientific Workshop on Biodegradable Plastics and Polymers, p. 261, Osaka, nov. (1994).

Enviado: $29 / 11 / 05$

Reenviado: 27/10/06

Aceito: 07/11/06 